\title{
Difference cordiality of product related graphs
}

\author{
R. Ponraj ${ }^{1}$, S. Sathish Narayanan ${ }^{1, *}$ and R. Kala ${ }^{2}$ \\ 1 Department of Mathematics, Sri Paramakalyani College, Alwarkurichi-627412, India \\ 2 Department of Mathematics, Manonmaniam Sundaranar University, Tirunelveli-627012, India \\ * Corresponding Author \\ E-mail: ponrajmaths@gmail.com, sathishrvss@gmail.com, karthipyi91@yahoo.co.in
}

\begin{abstract}
Let $G$ be a $(p, q)$ graph. Let $f: V(G) \rightarrow\{1,2, \ldots, p\}$ be a function. For each edge $u v$, assign the label $|f(u)-f(v)| . \quad f$ is called a difference cordial labeling if $f$ is an injective map and $\left|e_{f}(0)-e_{f}(1)\right| \leq 1$ where $e_{f}(1)$ and $e_{f}(0)$ denote the number of edges labeled with 1 and not labeled with 1 respectively. A graph which admits a difference cordial labeling is called a difference cordial graph. In this paper, we investigate the difference cordiality of torus grids $C_{m} \times C_{n}, K_{m} \times P_{2}$, prism, book, mobius ladder, Mongolian tent and $n$-cube.
\end{abstract}

2010 Mathematics Subject Classification. 05C78.

Keywords. Torus grids, Prism, Möbius ladder.

\section{Introduction}

Throughout this paper we have considered only simple and undirected graph. Let $G=(V, E)$ be a $(p, q)$ graph. The number $|V|$ is called the order of $\mathrm{G}$ and the number $|E|$ is called the size of $G$. The origin of the graph labeling problem is graceful labeling which was introduced by Rosa [11] in the year 1967. Ibrahim cahit [1] introduced the concept of cordial labeling in the year 1987. M. Sundaram, R. Ponraj and S. Somasundaram defined product cordial labeling of graphs. Cordial labeling and Product cordial labeling behavior of numerous graphs were studied by several authors $[2,12,13,14]$. On similar line, the notion of difference cordial labeling was introduced by R. Ponraj, S. Sathish Narayanan and R. Kala in [5]. In [5, 6, 7, 8, 9, 10], difference cordial labeling behavior of several graphs like path, cycle, complete graph, complete bipartite graph, bistar, wheel, web and some more standard graphs have been investigated. Here, we investigate the difference cordial labeling behavior of prism, Mongolian tent, book, young tableau, $K_{m} \times P_{2}$, torus grids, $n$-cube. Also we have proved that if $G_{1}$ and $G_{2}$ are $\left(p_{1}, q_{1}\right)$ and $\left(p_{2}, q_{2}\right)$ graphs with $q_{1} \geq p_{1}$ and $q_{2} \geq p_{2}$, then $G_{1} \times G_{2}$ is not difference cordial. Let $x$ be any real number. Then the symbol $\lfloor x\rfloor$ stands for the largest integer less than or equal to $x$ and $\lceil x\rceil$ stands for the smallest integer greater than or equal to $x$. Terms and definitions not defined here are used in the sense of Harary [4].

\section{Difference cordial labeling}

Definition 2.1. Let $G$ be a $(p, q)$ graph. Let $f$ be a map from $V(G)$ to $\{1,2 \ldots, p\}$. For each edge $u v$, assign the label $|f(u)-f(v)| . \quad f$ is called difference cordial labeling if $f$ is $1-1$ and $\left|e_{f}(0)-e_{f}(1)\right| \leq 1$ where $e_{f}(1)$ and $e_{f}(0)$ denote the number of edges labeled with 1 and not labeled with 1 respectively. A graph with a difference cordial labeling is called a difference cordial graph.

The following results (theorem 2.2 to 2.8 ) are used in the subsequent section. 
Theorem 2.2. [5] Any Path is a difference cordial graph.

Theorem 2.3. [5] Any Cycle is a difference cordial graph.

Theorem 2.4. [5] If $G$ is a $(p, q)$ difference cordial graph, then $q \leq 2 p-1$.

Theorem 2.5. [5] $K_{2, n}$ is difference cordial iff $n \leq 4$.

Theorem 2.6. [5] $K_{3, n}$ is difference cordial iff $n \leq 4$.

Theorem 2.7. [5] The wheel $W_{n}$ is difference cordial.

Theorem 2.8. [5] $K_{n}$ is difference cordial iff $n \leq 4$.

The product graph $G_{1} \times G_{2}$ is defined as follows: Consider any two points $u=\left(u_{1}, u_{2}\right)$ and $v=$ $\left(v_{1}, v_{2}\right)$ in $V=V_{1} \times V_{2}$. Then $u$ and $v$ are adjacent in $G_{1} \times G_{2}$ whenever $\left[u_{1}=v_{1}\right.$ and $u_{2}$ adj $\left.v_{2}\right]$ or $\left[u_{2}=v_{2}\right.$ and $u_{1}$ adj $\left.v_{1}\right]$.

Theorem 2.9. If $G_{1}$ and $G_{2}$ are $\left(p_{1}, q_{1}\right)$ and $\left(p_{2}, q_{2}\right)$ graphs respectively, with $q_{1} \geq p_{1}$ and $q_{2} \geq p_{2}$, then $G_{1} \times G_{2}$ is not difference cordial.

Proof. Clearly, $G_{1} \times G_{2}$ is a $\left(p_{1} p_{2}, p_{1} q_{2}+p_{2} q_{1}\right)$ graph. Suppose $G_{1} \times G_{2}$ is difference cordial. Then by theorem 2.4, $2 p_{1} p_{2}-1 \geq p_{1} q_{2}+p_{2} q_{1} \geq p_{1} p_{2}+p_{1} p_{2} \geq 2 p_{1} p_{2}$. This implies $-1 \geq 0$, a contradiction.

Q.E.D.

The graph $C_{m} \times C_{n}$ is called a Torus grid.

Corollary 2.10. Torus grids $C_{m} \times C_{n}$ are not difference cordial.

Prisms are graphs of the form $C_{m} \times P_{n}$. We now look into the graph prism $C_{n} \times P_{2}$.

Theorem 2.11. The prism $C_{n} \times P_{2}$ is difference cordial.

Proof. Let $V\left(C_{n} \times P_{2}\right)=\left\{u_{i}, v_{i}: 1 \leq i \leq n\right\}$ and $E\left(C_{n} \times P_{2}\right)=\left\{u_{1} u_{n}, v_{1} v_{n}\right\} \cup\left\{u_{i} v_{i}: 1 \leq i \leq n\right\} \cup$ $\left\{u_{i} u_{i+1}, v_{i} v_{i+1}: 1 \leq i \leq n-1\right\}$. Define a map $f: V\left(C_{n} \times P_{2}\right) \rightarrow\{1,2, \ldots, p\}$ as follows:

Case 1. $n$ is even.

Define $f\left(u_{1}\right)=1, f\left(u_{2}\right)=4, f\left(v_{1}\right)=2, f\left(v_{2}\right)=3$,

$$
\begin{aligned}
& f\left(u_{2 i+2}\right)=4 i+1, \quad 1 \leq i \leq \frac{n-2}{2} \\
& f\left(u_{2 i+1}\right)=4 i+4, \quad 1 \leq i \leq \frac{n-2}{2} \\
& f\left(v_{2 i+2}\right)=4 i+2, \quad 1 \leq i \leq \frac{n-2}{2} \\
& f\left(v_{2 i+1}\right)=4 i+3, \quad 1 \leq i \leq \frac{n-2}{2} .
\end{aligned}
$$

The following table 1 shows that $f$ is a difference cordial labeling.

\begin{tabular}{|c|c|c|}
\hline Nature of $n$ & $e_{f}(0)$ & $e_{f}(1)$ \\
\hline$n \equiv 0(\bmod 2)$ & $\frac{3 n}{2}$ & $\frac{3 n}{2}$ \\
\hline$n \equiv 1(\bmod 2)$ & $\frac{3 n+1}{2}$ & $\frac{3 n-1}{2}$ \\
\hline
\end{tabular}

TABLE 1.

Case 2. $n$ is odd.

Assign the labels to the vertices $u_{i}$ and $v_{i}(1 \leq i \leq n-1)$ as in case 1 . Define $f\left(u_{n}\right)=2 n$ and $f\left(u_{n-1}\right)=2 n-1$. Clearly, this labeling is a difference cordial labeling of $C_{n} \times P_{2}$ when $n$ is odd.

Q.E.D. 
Theorem 2.12. The graph $K_{m} \times P_{2}$ is difference cordial iff $m \leq 3$.

Proof. Since $K_{1} \times P_{2} \cong P_{2}$, by theorem 2.2, $K_{1} \times P_{2}$ is difference cordial. The graph $K_{2} \times P_{2} \cong C_{4}$. By theorem 2.3, $K_{2} \times P_{2}$ is difference cordial. $K_{3} \times P_{2}$ is a prism and hence difference cordial by theorem 2.11. Suppose $K_{m} \times P_{2}$ is difference cordial $(m>3)$. Clearly, $K_{m} \times P_{2}$ has $2 m$ vertices and $m^{2}$ edges. Using theorem $2.4, m^{2} \leq 4 m-1$. This is possible only when $m \leq 3$.

Q.E.D.

Theorem 2.13. Let $G$ be a $(p, q)$ connected graph. If $n \geq 5$, then $G \times K_{n}$ is not difference cordial.

Proof. The order and size of $G \times K_{n}$ are $n p$ and $n q+\left(\begin{array}{c}n \\ 2\end{array}\right) p$ respectively. Suppose $G \times K_{n}$ is difference cordial with $n \geq 5$. Then, by theorem $2.4, n q+\left(\begin{array}{c}n \\ 2\end{array}\right) p \leq 2 n p-1, \Rightarrow 5 n p-2 \geq 2 n q+n^{2} p \geq$ $2 n(p-1)+n^{2} p . \Rightarrow 8 \geq 10 p$, a contradiction.

Q.E.D.

Theorem 2.14. If $G$ is a $(p, q)$ connected graph. Then $G \times W_{n} \quad(n \geq 3)$ is difference cordial iff $p=1$.

Proof. The order and size of $G \times W_{n}$ are $(n+1) p$ and $2 n p+(n+1) q$ respectively. Suppose $G \times W_{n}$ is difference cordial with $p \geq 2$. Then, by theorem $2.4,2 n p+(n+1) q \leq 2(n+1) p-1 . \Rightarrow 2 p-1 \geq$ $(n+1) q \geq 4 q \geq 4(p-1)$. $\Rightarrow 3 \geq 2 p \geq 4$, a contradiction. When $p=1, G \cong K_{1}$. By theorem 2.7, $K_{1} \times W_{n} \cong W_{n}$ is difference cordial.

Q.E.D.

The book $B_{m}$ is the graph $S_{m} \times P_{2}$ where $S_{m}$ is the star with $m+1$ vertices.

Theorem 2.15. The book $B_{m}$ is difference cordial iff $m \leq 6$.

Proof. Let $V\left(B_{m}\right)=\left\{u, v, u_{i}, v_{i}: 1 \leq i \leq m\right\}$ and $E\left(B_{m}\right)=\left\{u v, u u_{i}, v v_{i}, u_{i} v_{i}: 1 \leq i \leq m\right\}$. For $m \leq 6$, the difference cordial labeling $f$ is given in table 2 .

\begin{tabular}{|c|c|c|c|c|c|c|c|c|c|c|c|c|c|c|}
\hline$n$ & $u$ & $v$ & $u_{1}$ & $u_{2}$ & $u_{3}$ & $u_{4}$ & $u_{5}$ & $u_{6}$ & $v_{1}$ & $v_{2}$ & $v_{3}$ & $v_{4}$ & $v_{5}$ & $v_{6}$ \\
\hline 2 & 4 & 3 & 1 & 6 & & & & & 2 & 5 & & & & \\
\hline 3 & 3 & 4 & 1 & 8 & 6 & & & & 2 & 7 & 5 & & & \\
\hline 4 & 4 & 3 & 1 & 5 & 7 & 9 & & & 2 & 6 & 8 & 10 & & \\
\hline 5 & 4 & 3 & 1 & 5 & 7 & 9 & 11 & & 2 & 6 & 8 & 10 & 12 & \\
\hline 6 & 4 & 3 & 1 & 5 & 7 & 9 & 11 & 13 & 2 & 6 & 8 & 10 & 12 & 14 \\
\hline
\end{tabular}

TABLE 2.

Suppose $m>6$. Let $f$ be a difference cordial labeling of $B_{m}$.

Claim: $e_{f}(1) \leq m+3$.

Case 1. Labels of $u$ and $v$ are consecutive numbers.

Let $f(u)=t$ and $f(v)=t+1$. There are at most two edges $u u_{i}$ and $v v_{j}$ with label 1 . The maximum value of $e_{f}(1)$ is attained when $u_{i}$ and $v_{i}$ receive the consecutive numbers. This forces $e_{f}(1) \leq m+1+2=m+3$.

Case 2. $f(u)$ is neither successor nor predecessor of $f(v)$.

In this case, there are at most four edges $u u_{i}$ and $v v_{j}$ with label 1 . Also, at least one of $u_{i} v_{i}(1 \leq i \leq m)$ receive the label 0 . Therefore, $e_{f}(1) \leq 4+m-1=m+3$. Hence, $e_{f}(0) \geq$ $q-(m+3) \geq 2 m-2$. This implies, $e_{f}(0)-e_{f}(1) \geq m-5 \geq 2$, a contradiction.

Q.E.D.

The graph $L_{n}=P_{n} \times P_{2}$ is called ladder. 
Theorem 2.16. Let $G$ be a graph obtained from a ladder $L_{n}$ by subdividing each step exactly once. Then $G$ is difference cordial.

Proof. Let $V(G)=\left\{u_{i}, v_{i}, w_{i}: 1 \leq i \leq n\right\}$ and $E(G)=\left\{u_{i} w_{i}, w_{i} v_{i}: 1 \leq i \leq n\right\} \cup\left\{u_{i} u_{i+1}, v_{i} v_{i+1}\right.$ : $1 \leq i \leq n-1\}$. Define a map $f: V(G) \rightarrow\{1,2 \ldots 3 n\}$ by $f\left(u_{i}\right)=i, 1 \leq i \leq n, f\left(v_{i}\right)=n+1+i, 1 \leq$ $i \leq n, f\left(w_{i}\right)=2 n+1+i, 1 \leq i \leq n-1, f\left(w_{n}\right)=n+1$. Since $e_{f}(0)=e_{f}(1)=2 n-1, f$ is a difference cordial labeling of $G$.

Q.E.D.

Next is the Möbius ladder. The Möbius ladder $M_{n}$ is the graph obtained from the ladder $L_{n}$ by joining the opposite end vertices of two copies of $P_{n}$.

Theorem 2.17. The Möbius ladder $M_{n}$ is difference cordial.

Proof. Let $V\left(M_{n}\right)=\left\{u_{i}, v_{i}: 1 \leq i \leq n\right\}$ and $E\left(M_{n}\right)=\left\{u_{i} v_{i}: 1 \leq i \leq n\right\} \cup\left\{u_{i} u_{i+1}, v_{i} v_{i+1}: 1 \leq\right.$ $i \leq n-1\} \cup\left\{u_{1} v_{n}, v_{1} u_{n}\right\}$. Clearly, $M_{n}$ consists of $2 n$ vertices and $3 n$ edges.

Case 1. $n$ is even.

Define a map $f: V\left(M_{n}\right) \rightarrow\{1,2 \ldots 2 n\}$ by

$$
\left.\begin{array}{lll}
f\left(u_{2 i-1}\right)=4 i-3, & 1 \leq i \leq\left[\begin{array}{c}
\frac{n}{2} \\
f\left(u_{2 i}\right)
\end{array}=4 i-2,\right. & 1 \leq i \leq\left[\frac{n}{2}\right. \\
f\left(v_{2 i-1}\right) & =4 i, & 1 \leq i \leq\left[\frac{n}{2}\right. \\
f\left(v_{2 i}\right) & =4 i-1, & 1 \leq i \leq\left[\frac{n}{2}\right.
\end{array}\right]
$$

Case 2. $n$ is odd.

Label the vertices $u_{2 i-1}\left(1 \leq i \leq\left\lceil\frac{n}{2}\right\rceil\right)$ and $u_{2 i}, v_{2 i-1}, v_{2 i}\left(1 \leq i \leq\left\lfloor\frac{n}{2}\right\rfloor\right)$ as in case 1 and define $f\left(v_{n}\right)=2 n$. The following table 3 proves that $f$ is a difference cordial labeling of $M_{n}$

\begin{tabular}{|c|c|c|}
\hline Nature of $n$ & $e_{f}(0)$ & $e_{f}(1)$ \\
\hline$n \equiv 0(\bmod 2)$ & $\frac{3 n}{2}$ & $\frac{3 n}{2}$ \\
\hline$n \equiv 1(\bmod 2), n \neq 3$ & $\frac{3 n+1}{2}$ & $\frac{3 n-1}{2}$ \\
\hline$n=3$ & 4 & 5 \\
\hline
\end{tabular}

TABle 3.

Q.E.D.

A Young tableau is a sub graph of $P_{m} \times P_{n}$ obtained by retaining the first two rows of $P_{m} \times P_{n}$ and deleting the vertices from the right hand end of other rows in such a way that the lengths of the successive rows form a non increasing sequence.

Theorem 2.18. Let $G$ be a graph obtained from a Young tableau which is obtained from the grid $P_{n} \times P_{n} \quad(n$ odd $)$, by adding an extra vertex above the top row of a Young tableau and joining every vertex of the top row to the extra vertex. Then $G$ is difference cordial.

Proof. Consider the right corner vertex of the top row. Label that vertex by 1 . Then assign the labels $2,3 \ldots n$ to the preceding vertices of the top row. That is, the left corner vertex of the top row receive the label $n$. Then we move to the second row. Assign the label $n+1$ to the left corner vertex of the second row. Then assign the labels $n+1, n+2, \ldots 2 n$ to the successive vertices of 
the second row. Then we move to the right corner vertex of the third row and label it by $2 n+1$ and the preceding vertices of third row are labeled by $2 n+2,2 n+3, \ldots 3 n-1$. Then we move to the left corner vertex of the fourth row and so on. Finally, assign the label $p$ to the extra vertex. Obviously, this vertex labeling is difference cordial labeling.

Q.E.D.

A difference cordial labeling of $G$ with $n=7$ is given in figure 1 .

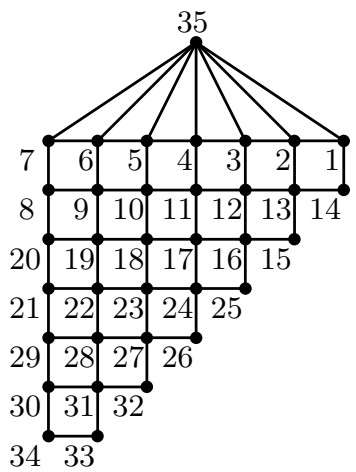

\section{FIGURE 1.}

A Mongolian tent $M T_{m, n}$ is a graph obtained from $P_{m} \times P_{n}, n$ odd, by adding one extra vertex above the grid and joining every other vertex of the top row of $P_{m} \times P_{n}$ to the new vertex.

Theorem 2.19. The Mongolian tent $M T_{m, n}$ ( $n$ odd) is difference cordial.

Proof. Let $V\left(M T_{m, n}\right)=\left\{u, u_{i, j}: 1 \leq i \leq m, 1 \leq j \leq n\right\}$ and $E\left(M T_{m, n}\right)=\left\{u u_{i, j}: 1 \leq i \leq m, 1 \leq\right.$ $j \leq n-1\} \cup\left\{u_{i, j} u_{i, j+1}: 1 \leq i \leq m, 1 \leq j \leq n-1\right\} \cup\left\{u_{i, j} u_{i+1, j}: 1 \leq i \leq m-1,1 \leq j \leq n\right\}$. The order and size of $M T_{m, n}$ are $m n+1$ and $2 m n-m$ respectively. Define an injective map $f$ from the vertices of $M T_{m, n}$ to the set $\{1,2 \ldots, m n+1\}$ as follows:

$$
\begin{aligned}
& f\left(u_{4 i-3,1}\right)=4 n(i-1)+1 \quad 1 \leq i \leq \frac{m}{4} \quad \text { if } \quad m \equiv 0(\bmod 4) \\
& 1 \leq i \leq \frac{m+3}{4} \quad \text { if } \quad m \equiv 1(\bmod 4) \\
& 1 \leq i \leq \frac{m+2}{4} \quad \text { if } \quad m \equiv 2(\bmod 4) \\
& 1 \leq i \leq \frac{m+1}{4} \quad \text { if } \quad m \equiv 3(\bmod 4) \text {. } \\
& f\left(u_{4 i-2,1}\right)=(4 i-2) n \quad 1 \leq i \leq \frac{m}{4} \quad \text { if } \quad m \equiv 0(\bmod 4) \\
& 1 \leq i \leq \frac{m-1}{4} \quad \text { if } \quad m \equiv 1(\bmod 4) \\
& 1 \leq i \leq \frac{m+2}{4} \quad \text { if } \quad m \equiv 2(\bmod 4) \\
& 1 \leq i \leq \frac{m+1}{4} \quad \text { if } \quad m \equiv 3(\bmod 4) \text {. } \\
& f\left(u_{4 i-1,1}\right)=(4 i-1) n \quad 1 \leq i \leq \frac{m}{4} \quad \text { if } \quad m \equiv 0(\bmod 4) \\
& 1 \leq i \leq \frac{m-1}{4} \quad \text { if } \quad m \equiv 1(\bmod 4) \\
& 1 \leq i \leq \frac{m-2}{4} \quad \text { if } \quad m \equiv 2(\bmod 4) \\
& 1 \leq i \leq \frac{m+1}{4} \quad \text { if } \quad m \equiv 3(\bmod 4) \text {. } \\
& f\left(u_{4 i, 1}\right)=n(4 i-1)+1 \quad 1 \leq i \leq \frac{m}{4} \quad \text { if } \quad m \equiv 0(\bmod 4) \\
& 1 \leq i \leq \frac{m-1}{4} \quad \text { if } \quad m \equiv 1(\bmod 4) \\
& 1 \leq i \leq \frac{m-2}{4} \quad \text { if } \quad m \equiv 2(\bmod 4) \\
& 1 \leq i \leq \frac{m-3}{4} \quad \text { if } \quad m \equiv 3(\bmod 4) \text {. }
\end{aligned}
$$




$$
\begin{aligned}
& f\left(u_{4 i-3, j}\right)=f\left(u_{4 i-3, j-1}\right)+1 \quad 2 \leq j \leq n \\
& 1 \leq i \leq \frac{m}{4} \quad \text { if } \quad m \equiv 0(\bmod 4) \\
& 1 \leq i \leq \frac{m+3}{4} \quad \text { if } \quad m \equiv 1(\bmod 4) \\
& 1 \leq i \leq \frac{m+2}{4} \quad \text { if } \quad m \equiv 2(\bmod 4)
\end{aligned}
$$

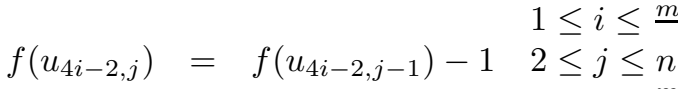

$$
\begin{aligned}
& 1 \leq i \leq \frac{m}{4} \quad \text { if } \quad m \equiv 0(\bmod 4) \\
& 1 \leq i \leq \frac{m-1}{4} \quad \text { if } \quad m \equiv 1(\bmod 4) \\
& 1 \leq i \leq \frac{m+2}{4} \quad \text { if } \quad m \equiv 2(\bmod 4) \\
& f\left(u_{4 i-1, j}\right)=f\left(u_{4 i-1, j-1}\right)-1 \quad 2 \leq j \leq n \\
& 1 \leq i \leq \frac{m}{4} \quad \text { if } \quad m \equiv 0(\bmod 4) \\
& 1 \leq i \leq \frac{m-1}{4} \quad \text { if } \quad m \equiv 1(\bmod 4) \\
& 1 \leq i \leq \frac{m-2}{4} \quad \text { if } \quad m \equiv 2(\bmod 4) \\
& f\left(u_{4 i, j}\right)=f\left(u_{4 i, j-1}\right)+1 \quad 2 \leq j \leq n \\
& 1 \leq i \leq \frac{m+1}{4} \quad \text { if } \quad m \equiv 3(\bmod 4) \text {. } \\
& 1 \leq i \leq \frac{m}{4} \quad \text { if } \quad m \equiv 0(\bmod 4) \\
& 1 \leq i \leq \frac{m-1}{4} \quad \text { if } \quad m \equiv 1(\bmod 4) \\
& 1 \leq i \leq \frac{m-2}{4} \quad \text { if } \quad m \equiv 2(\bmod 4) \\
& 1 \leq i \leq \frac{m-3}{4} \quad \text { if } \quad m \equiv 3(\bmod 4) .
\end{aligned}
$$

and $f(u)=m n+1$. The following table 4 shows that $f$ is a difference cordial labeling of the Mongolian tent $M T_{m, n}$.

\begin{tabular}{|c|c|c|}
\hline Nature of $m$ & $e_{f}(0)$ & $e_{f}(1)$ \\
\hline$m \equiv 0(\bmod 2)$ & $\frac{2 m n-m}{2}$ & $\frac{2 m n-m}{2}$ \\
\hline$m \equiv 1(\bmod 2)$ & $\frac{2 m n-m+1}{2}$ & $\frac{2 m n-m-1}{2}$ \\
\hline
\end{tabular}

TABLE 4.

Q.E.D.

Finally, we investigate the $n$-cube.

Theorem 2.20. $K_{2} \times K_{2} \times \cdots \times K_{2} \quad(n$ times $)$ is difference cordial.

Proof. Let $G=K_{2} \times K_{2} \times \ldots \times K_{2}$ (n times). Let $V(G)=\left\{u_{i}, v_{i}, w_{i}, x_{i}: 1 \leq i \leq n-1\right\}$ and $E(G)=\left\{u_{i} v_{i}, v_{i} x_{i}, x_{i} w_{i}, w_{i} u_{i}: 1 \leq i \leq n-1\right\} \cup\left\{u_{i} u_{i+1}, v_{i} v_{i+1}, w_{i} w_{i+1}, x_{i} x_{i+1}: 1 \leq i \leq n-2\right\}$. Define a map $f: V(G) \rightarrow\{1,2, \ldots, 4 n-4\}$ by $f\left(u_{i}\right)=i, 1 \leq i \leq n-1, f\left(v_{n-i+1}\right)=n-1+i$, $1 \leq i \leq n-1, f\left(w_{i}\right)=2 n-2+i, 1 \leq i \leq n-1, f\left(x_{n-i+1}\right)=3 n-3+i, 1 \leq i \leq n-1$. Since $e_{f}(0)=e_{f}(1)=4 n-6, f$ is a difference cordial labeling of $G$.

Q.E.D.

\section{References}

[1] I. Cahit, Cordial graphs: a weeker version of graceful and harmonious graphs, Ars Combin., 23 (1987), 201-207. 
[2] E. Salehi, PC-labelings of a graphs and its PC-sets, Bull. Inst. Combin. Appl., 58 (2010), 112121.

[3] J. A. Gallian, A Dynamic survey of graph labeling, The Electronic Journal of Combinatorics, 18 (2012), \#Ds6.

[4] F. Harary, Graph theory, Addision wesley, New Delhi (1969).

[5] R. Ponraj, S. Sathish Narayanan and R. Kala, Difference cordial labeling of graphs, Global Journal of Mathematical Sciences: Theory and Practical, 5 (2013), 185-196.

[6] R. Ponraj, S. Sathish Narayanan and R.Kala, Difference cordial labeling of corona graphs, J. Math. Comput. Sci., 3(2013), 1237-1251.

[7] R. Ponraj and S. Sathish Narayanan, Difference cordial labeling of some derived graphs, International journal of Mathematical combinatorics, 4 (2014), 37-48.

[8] R. Ponraj and S. Sathish Narayanan, Difference cordial labeling of some snake graphs, Journal of Applied Mathematics and Informatics, 32(3-4) (2014), 377-387.

[9] R. Ponraj, S. Sathish Narayanan and R. Kala, A note on difference cordial graphs, Palestein Journal of Mathematics, 4(1) (2015), 189-197.

[10] R. Ponraj and S. Sathish Narayanan, Difference cordial labeling of graphs obtained from triangular snakes, Application and Applied Mathematics, 9(2) (2014), 811-825.

[11] A. Rosa, On certain valuation of the vertices of a graph, Theory of Graphs (Internat. Symposium, Rome, July 1966), Gordon and Breach, N. Y. and Dunod Paris (1967), 349-355.

[12] M. Z. Youssef, On Skolem-graceful and cordial graphs, Ars Combin., 78 (2006), 167-177.

[13] M. Z. Youssef, On k-cordial labelling, Australas. J. Combin., 43 (2009), 31-37.

[14] M. Z. Youssef, Graph operations and cordiality, Ars Combin., 97 (2010), 161-174. 